\title{
Random Walk and Lighting Control
}

\author{
Matthew Aldrich, Akash Badshah, Brian Mayton, Nan Zhao, Joseph A. Paradiso \\ Responsive Environments Group, MIT Media Lab \\ Massachusetts Institute of Technology, Cambridge, MA 02139 \\ Email: \{maldrich,akashbad,bmayton,nanzhao,joep\}@media.mit.edu
}

\begin{abstract}
We pose the problem of turning off a single luminaire (or group) as an optimal stopping problem. We present the stationary and first-passage analysis of motion data obtained using custom wireless nodes in an open office floor plan. These calculations allow us to estimate the state of the network and calculate the probability and expected number of steps to visit a state from any arbitrary state. We also investigate if there is any evidence of clustering amongst the nodes by studying the covariance of the dataset. The data indicate the existence of clustering within the lattice. In other words, the analysis of random walk prevents luminaires from accidentally shutting off and dimensionality reduction determines the correct zoning of lighting via the occupants' movements.
\end{abstract}

\section{INTRODUCTION}

The requisite building blocks of our emerging "digital nervous system" are now in place. At the heart of this cyberphysical infrastructure, we find collections of sensors and a myriad of applications that are interwoven in our buildings, homes, and daily life. Looking beyond Weiser's vision of ubiquitous computing [1], the road ahead not only requires innovations in silicon and sensing, but also commands that our community take an earnest look at the human facet of our rapidly evolving and ever-scaling "invisible network." As a species, we are not yet "plugging" into our digital nervous system, but we are acknowledging its presence (see Figure 1). But mere visualization of the pure sensor phenomena may lack critical information for building designers, technicians, engineers, and the public. This is the divide between data and information.

These concerns are partially addressed in this work. Our primary study focuses around common building technology and observing what happens when the network is studied as a whole. While our goal is studying occupancy-related phenomena to improve lighting control in open floor plans, the techniques employed and subsequent results are general.

\section{A. Lighting}

Motion sensors are a common and preferred method of lighting control in open-office floor plans. While the motion sensors are excellent at automatically turning on the lights in the room, lack of motion and temporary occlusion can lead to the lights being unexpectedly turned off (a false-negative, or type II error). It is reasonable to assume that in the near future, multiple-sensor functionality will be included in the officeplan lighting; perhaps increasing the density of motion sensor coverage on the floor. How can this dense motion-sensor array

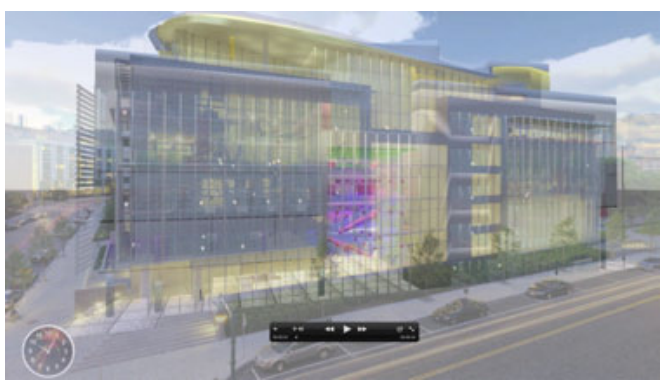

Fig. 1: The Responsive Environments group's "Doppellab" enables spatio-temporal browsing of live sensor data in a virtual 3D environment [9].

be utilized to minimize type II errors in the lighting control network?

Examples of lighting and sensor networks found in [2], [3], [4], [5], demonstrate that sensor networks and lighting are inseparable. However, deterministic properties of control using these approaches are entirely at odds with our human notions of aesthetics and appearance. Our deterministic views of lighting control, for instance, that "the light here should be this bright and minimize the energy to do so," represent a tractable problem in optimization, but in reality, fail to account for anything other than the surface illuminance and energy consumption.

To date, occupancy-based controls represent the majority of automatic building lighting control systems. Early studies of stochastic modeling and lighting are reported in [6], [7], [8]. In this work, we study the effects of a network of dense occupancy sensors, under the assumption that future networked-lighting control systems (inherently digital) will contain a multitude of sensing capabilities.

\section{BACKGROUND}

\section{A. Markov Processes}

The concept of random walk arises in this analysis because of our use of Markov chains to analyze both the stationary (i.e., equilibrium behavior) and dynamic (i.e., absorption probabilities and hitting times) properties of the observed sensor data.

A Markov process $\left\{X_{t}\right\}$ is a stochastic process with the property that, given the value of $X_{t}$, the values of $X_{s}$ for $s>t$ are not influenced by the values of $X_{u}$ for $u<t$. In other words, the probability of any particular future behavior of the process, when its current state is known exactly, is not 
altered by additional knowledge concerning its past behavior [10]

In this work, we focus primarily on the resulting description of the sensor phenomena modeled using stochastic processes. For an introduction to Markov chains (and other stochastic analysis) consult [10]. A greater mathematical emphasis is given in [11] but, applications are still emphasized. Lightingspecific use of occupancy is reviewed in [12]. Alternatively, one may study these phenomena within the framework of Brownian motion and normal distributions ${ }^{1}$.

Our analysis allows one to calculate the expected time to randomly walk from any arbitrary state to a state of interest. Furthermore, one can calculate the chances of doing so. These properties have very clear interpretations in lighting control. The hitting time characterizes the optimal stopping time in which the light (or lights) can be switched off. In effect, this analysis provides a mathematical framework for guaranteeing the optimality of luminaire-specific timers that turn the lights off. Of course, an entirely different stopping problem may also exist, see [13] for an overview.

What this framework subtly suggests is that these models can be implemented at the per-node scale and subsequently, lighting networks (and controls) might represent autonomous agents, capable of making their own decisions without the overhead of additional computing.

\section{B. Complex Networks}

These sensor nodes can be shown to represent a complex network. Although their state is driven by purely exogenous inputs (our movement), our positions within this network are not entirely as random as a Markovian approach suggests (an Erdôs-Rényi network, for example, permit the study of purely random phenomena). The primary issue with these random networks is that, by definition, there is no community structure (a concept in network science that allows grouping of similar nodes, see [14]). While this fact is true of Erdős-Rényi networks, this not specifically the case in occupancy networks (e.g., it's impossible for a single person to disappear and reappear in a different part of the network).

The random error in measurement is introduced because the occupancy data are unlabeled (simultaneous transition in multiple positions of the network due to multiple inhabitants are ambiguous). These types of problems, specifically for tracking individuals using occupancy data, are studied in [15].

Yet, over time, specific patterns emerge. One can study either the covariance or correlation matrix of the sensor nodes and perform dimensionality reduction (either using principal component analysis or factor analysis, resp.) to study if clustering is possible. These are so-called dimensionality reduction techniques.

\footnotetext{
${ }^{1}$ In this case, the sensor data can be modeled using Brownian motion with drift, $X(t)=\mu t+\sigma B(t)$. The drift parameter $\mu$ arises due to imbalances of the random walk. In the analysis that follows, the observed probabilities imply that drift is present (e.g., for two states $\neq \frac{1}{2}$ ).
}

\section{METHOD}

Thirty-four sensors nodes were deployed on the fifth floor of the MIT Media Lab (approx. $18 \times 16 \mathrm{~m}^{2}$ ) for an eightmonth period in order to observe occupancy (see floor plan in Figure 2). This lab area houses three distinct research groups and a common electronics prototype area. The sensor nodes collected occupancy data and illuminance data every 30 seconds and transmit these data back to a computer for logging.

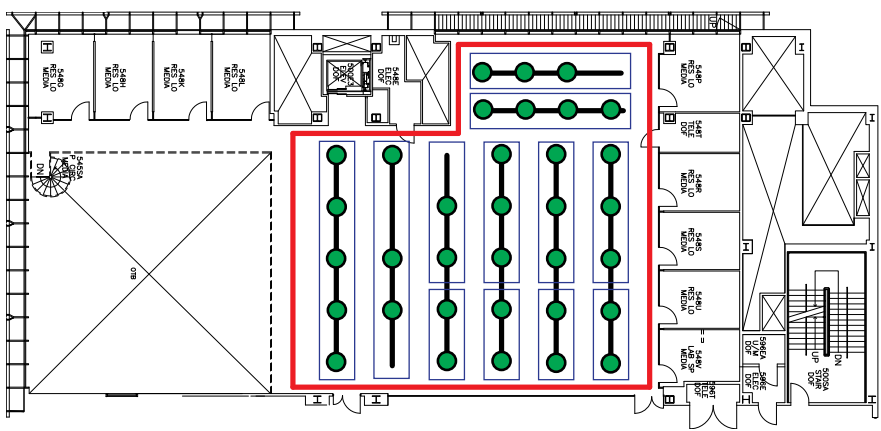

Fig. 2: The sensor installation plan (red). The green nodes represent the sensors. Blue rectangles indicate luminaire zones.

We customer-developed the sensor node at the MIT Media Lab for the purpose of studying lighting-related phenomena in large open-scale floor plans. Each node is comprised of an ATmega168 MCU, an AT86RF230 802.15 radio (both Atmel parts) and an AMN11112 low-power PIR sensor (Panasonic), related power and sensor conditioning circuity, and is powered by a $9 \mathrm{~V}$ battery (see Figure 3 ). The expected operating time of the device is approximately 8 to 12 months.

The devices are mounted magnetically to the brackets of fluorescent lighting system in the Media Lab. Care was taken to create a network (technically, a lattice) that was as regular as possible, yet architectural constraints impacted this goal.

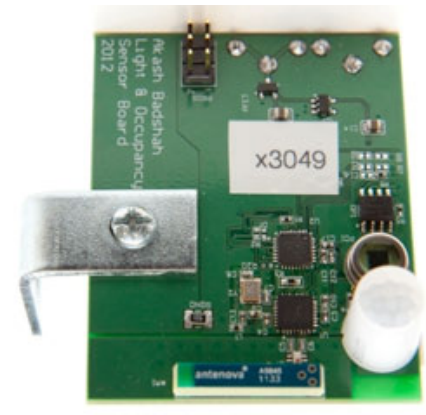

(a)

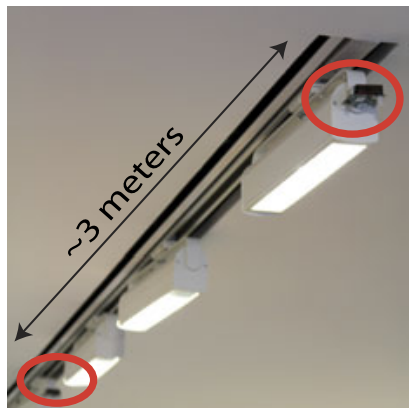

(b)
Fig. 3: In (a), the custom sensor node used in the experiments. In (b), the magnetic attachment of the sensors (circled in red) and the approximate distance between the nodes.

\section{RESUlts}

In this section, we present the results of studying movement and occupancy in the network as a Markov process for several 
TABLE I: Measured parameters for a two-state Markov process.

\begin{tabular}{lrrrr}
\hline SensorID & $\pi_{0}$ & $\pi_{1}$ & $E\left[T \mid X_{1}\right]$ & $E\left[T \mid X_{0}\right]$ \\
\hline 1 & 0.897 & 0.103 & 3 & 24 \\
2 & 0.899 & 0.101 & 4 & 34 \\
3 & 0.980 & 0.020 & 3 & 131 \\
4 & 0.743 & 0.257 & 3 & 8 \\
5 & 0.780 & 0.220 & 3 & 9 \\
6 & 0.745 & 0.255 & 3 & 8 \\
7 & 0.854 & 0.146 & 2 & 12 \\
8 & 0.710 & 0.290 & 3 & 8 \\
9 & 0.678 & 0.322 & 4 & 7 \\
10 & 0.747 & 0.253 & 3 & 9 \\
\hline Floor & 0.434 & 0.566 & 12 & 9 \\
\hline
\end{tabular}

TABLE II: Measured parameters for a four-state Markov process.

\begin{tabular}{lrrrrrr}
\hline SensorID & $\pi_{0}$ & $\pi_{1}$ & $\pi_{2}$ & $\pi_{3}$ & $E\left[T \mid X_{3}\right]$ & $E\left[T \mid X_{0}\right]$ \\
\hline 1 & 0.855 & 0.038 & 0.038 & 0.069 & 6 & 57 \\
2 & 0.869 & 0.027 & 0.027 & 0.076 & 7 & 70 \\
3 & 0.972 & 0.008 & 0.008 & 0.013 & 6 & 328 \\
4 & 0.653 & 0.089 & 0.089 & 0.170 & 7 & 21 \\
5 & 0.703 & 0.084 & 0.084 & 0.130 & 6 & 26 \\
6 & 0.652 & 0.090 & 0.090 & 0.167 & 7 & 21 \\
7 & 0.783 & 0.069 & 0.069 & 0.079 & 5 & 36 \\
8 & 0.615 & 0.089 & 0.089 & 0.207 & 8 & 20 \\
9 & 0.572 & 0.094 & 0.094 & 0.241 & 9 & 18 \\
10 & 0.660 & 0.083 & 0.083 & 0.174 & 7 & 23 \\
\hline Floor & 0.384 & 0.046 & 0.046 & 0.523 & 25 & 19 \\
\hline
\end{tabular}

configurations. Next, using principal component analysis, we study the network covariance matrix and present the visual result of the $2 \mathrm{D}$ transition probabilities. The data are presumed to be synchronous and the calculations are based on a fiveminute window.

\section{A. Results: Markov Processes}

The transition probabilities of a single node being "occupied" versus "not occupied" were calculated from a sequence of $1 \mathrm{~s}$ and 0s obtained via the sensors (repeated Bernoulli trials). The equilibrium state and hitting times were then calculated. These data (abridged) are shown in Table I and Table II for the first ten nodes and the entire network (see the Appendix for the full tables). ${ }^{2}$ The entire network category, "floor" is calculated by OR'ing all 34 readings; this category represents the "worst-case" category. These readings were obtained using five-minute synchronous intervals.

In Table I, the calculated equilibrium states $\pi_{i}$ are listed. These values correspond to the long-run behavior of the "unoccupied" and "occupied" states. This implies, for example, that sensor 10 is unoccupied $75 \%$ of the time, and occupied $25 \%$ of the time. As expected, these readings are different across the 34 nodes, and sensors positioned over desk areas produce different readings than those above foot-paths. Of note

\footnotetext{
${ }^{2}$ The tables containing the calculations for all 34 nodes are hosted online at http://media.mit.edu/resenv/lighting/sensors 13
}

is sensor 3, suggesting poor initial placement of the node (it is in a low-traffic area).

Still focusing on Table I, the hitting-times, $E\left[T \mid X_{i}\right]$ are calculated. Recall that these calculations are directly related to the time required to shut-off the luminaire. The column $E\left[T \mid X_{1}\right]$ is read as "starting in $X_{1}$ (the occupied state), the mean time until being absorbed by $X_{0}$ is $n$ steps." According to the data, this is roughly 3 to 4 steps for the sensors reported. If the timescale per step is 5 minutes, then the calculated shutoff time no greater than 20 minutes from the last observed "occupied" reading.

In Table II, a second-order Markov chain was studied. In this case, the current state depended on the two prior states (which may be 1 of 4 possible combinations of "occupied" and "unoccupied"). These two inner states $\pi_{1}$ and $\pi_{2}$ model some of the dynamics observed in the network (e.g., transitory activity). The table is interpreted the same as the previous table and, we note the primary effect of the second-order model is an increased turn-off time.

\section{B. Results: Clustering of Nodes Using Principal Components}

Principal component analysis was applied to a centered and scaled covariance matrix obtained using over $60 \times 10^{3}$ observations. We summarize these results in Figure 4. The sensor locations were known apriori, but the node colors in the figure represent the resulting clusters in the analysis. These distinct divisions correspond to the specific locations of 3 research groups and one common soldering area, hence reflect natural destinations. The transition probabilities between the nodes are also shown.

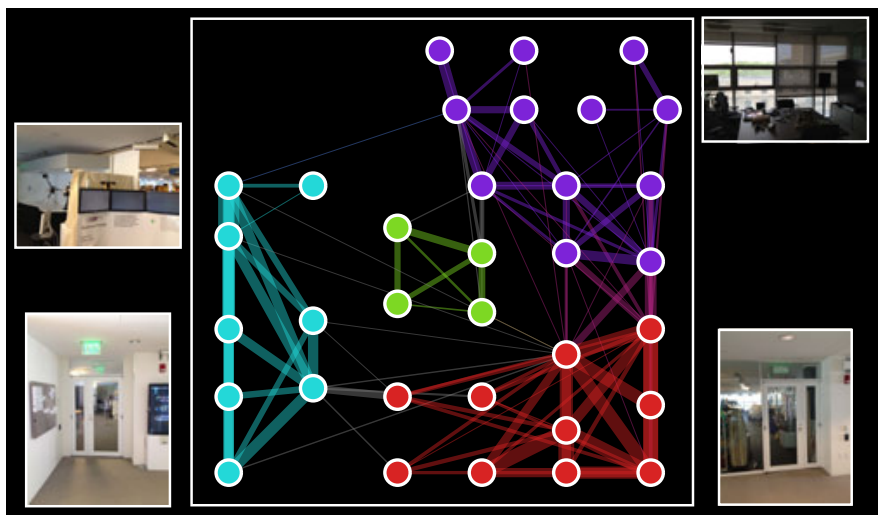

Fig. 4: The 34 sensor nodes, when projected onto their principal components, cluster. The clusters are color-coded and the node location is known apriori. The thickness is proportional to the transition probability.

\section{Discussion}

\section{A. Markov Processes}

The analysis of the Markov chains provides clear evidence that prior behavior can be used to improve automatic lighting control. The framework discussed here allows one to infer the optimal timer settings in a control system. The key point is that 
the "timer" does not start until the first zero is detected. Given the findings, it appears that 15 minutes is considered optimal in this building according to one model. A second-order model approximately doubles this time (see Table II). Interestingly, across the entire network, readings are approximately 4 times greater than those of a single node. This finding suggests that timer-settings in low density sensor installations may be inappropriately low.

Of course, since occupancy is not uniform over the entire lattice, the benefits of increased resolution and sensing allow the nodes to function independently of each other. The equilibrium states provide a clear indication of how much power can be saved utilizing these switching schemes. The observed potential for energy savings, according to the most conservative data, is between $38 \%$ and $43 \%$.

Yet, safety concerns and occupant comfort remain important, in lieu of energy minimization. For example, consider the parameter $E\left[T \mid X_{0}\right]$. Its direct interpretation is the expected time until the light is switched back on. In other words, both hitting-time parameters taken together give an indication of how many "on-off" cycles may occur in the network per hour. Of course, this can be mitigated by introducing some scaling factor, say $\beta /(1-\beta)$.

\section{B. Clustering in the Occupancy Network}

It is pleasing to find evidence that the nodes and related occupancy readings cluster within the boundaries of the research groups on this floor. In many ways, this may be an expected result. Human movement is deliberate within the space and tends to occur in repetitions, often in local loops. This group phenomena is also affected by the architectural arrangement and other fixed obstacles in the space. There exist only so many ways of approaching your desk, the soldering area, etc. It's possible that these clusterings may shift over time, due to architectural rearrangements, new people, and different social relationships between the research groups.

Alternatively, this result and the subsequent clustering may not be expected if one assumes that the shared space is used communally. In effect, these "invisible borders" indicate just the opposite; they possibly demonstrate the lack of collaboration (and interaction) between the groups. This is a critical area that may have applications in understanding sentiment and attitudes of different groups in large offices. For example, it is possible that this same experiment could be replicated in a different area, with different people, and building arrangements and find that no separation exists.

Regardless of what causes the observed phenomena, the results suggest that present zoning controls are inadequate based on how we actually use the space. These results suggest that our movement and use of the space are indicators of the natural boundaries of the lighting network. Another interpretation is that zoning of lighting is a natural process that depends on the inhabitants and their collective use of the space; it is not entirely reflected or captured through the experience of the lighting designer or building technician.

\section{CONCLUSIONS}

The application of Markov chains and lighting control is presented. We discuss how the subsequent analysis is an appropriate framework to derive optimal parameters of control in large-scale building floor plans and address a frustrating topic for building dwellers: how to turn the lights off. The results suggest that multi-scale sensor observations can reduce wasted lighting costs and mitigate fixed-timer building controls. Nonetheless, safety and comfort are critical factors in future work. An obvious starting point is to test the control of the network using the information learned in the study. Overall, these results lead us to believe that automatic learning and optimization of lighting control parameters are possible and, offers a worthwhile goal for next-generation lighting systems.

Additionally, we present a study of group patterns of occupancy. We find, in our case, evidence that movement and occupancy are confined to the invisible "borders" between research groups, perhaps induced by naturally occurring boundaries; possibly induced by lack of interaction between the research groups.

\section{REFERENCES}

[1] M. Weiser, "The computer for the 21st century," Scientific American, vol. 265, no. 3, pp. 94-104, 1991.

[2] D. Hunt, "Predicting artificial lighting use-a method based upon observed patterns of behaviour," Lighting research and technology, vol. 12, no. 1 , p. 7, 1980.

[3] Y. Wen, J. Granderson, and A. Agogino, "Towards embedded wirelessnetworked intelligent daylighting systems for commercial buildings," in Sensor Networks, Ubiquitous, and Trustworthy Computing, 2006. IEEE International Conference on, vol. 1. IEEE, 2006, pp. 6-pp.

[4] M. Aldrich, N. Zhao, and J. Paradiso, "Energy efficient control of polychromatic solid state lighting using a sensor network," vol. 7784, no. 1. SPIE, 2010, p. 778408.

[5] D. Caicedo, A. Pandharipande, and G. Leus, "Occupancy-based illumination control of led lighting systems," Lighting Research and Technology, vol. 43, no. 2, pp. 217-234, 2011.

[6] G. Newsham, A. Mahdavi, I. Beausoleil-Morrison, et al., "Lightswitch: a stochastic model for predicting office lighting energy consumption," in Proceedings of Right Light Three, the 3rd European Conference on Energy Efficient Lighting, 1995, pp. 60-66.

[7] C. Reinhart, "Lightswitch-2002: a model for manual and automated control of electric lighting and blinds," Solar Energy, vol. 77, no. 1, pp. 15-28, 2004.

[8] V. Singhvi, A. Krause, C. Guestrin, J. H. Garrett, Jr., and H. S. Matthews, "Intelligent light control using sensor networks," in Proc. Sensys'05. New York, NY, USA: ACM, 2005, pp. 218-229.

[9] G. Dublon, L. Pardue, B. Mayton, N. Swartz, N. Joliat, P. Hurst, and J. Paradiso, "Doppellab: Tools for exploring and harnessing multimodal sensor network data," in Sensors, 2011 IEEE, oct. 2011, pp. $1612-1615$.

[10] M. Pinsky and S. Karlin, An introduction to stochastic modeling. Academic press, 2010.

[11] S. I. Resnick, Adventures in stochastic processes. Springer, 1992.

[12] X. Guo, D. Tiller, G. Henze, and C. Waters, "The performance of occupancy-based lighting control systems: A review," Lighting Research and Technology, vol. 42, no. 4, pp. 415-431, 2010.

[13] "Optimal stopping and applications," http://www.math.ucla.edu/ tom/Stopping/Contents.html, accessed: 2013-08-01.

[14] M. Newman, "Modularity and community structure in networks," Proceedings of the National Academy of Sciences, vol. 103, no. 23, pp. 8577-8582, 2006.

[15] Y. Ivanov, E. Sorokin, C. Wren, I. Kaur, Y. I. A, E. S. B, C. W. A, and I. K. C, "Tracking people in mixed modality systems," in In Visual Communications and Image Processing, volume EI123. IS\&T/SPIE, 2007. 